\title{
Crystalline-Crystalline Block Copolymers of Regioregular Poly(3- hexylthiophene) and Polyethylene by Ring-Opening Metathesis Polymerization
}

\author{
Christopher P. Radano, ${ }^{\dagger}$ Oren A. Scherman, ${ }^{\dagger}$ Natalie Stutzmann-Stingelin, ${ }^{\S}$ Christian \\ Müller, ${ }^{\ddagger}$ Dag W. Breiby, Paul Smith, ${ }^{\ddagger}$ René A. J. Janssen, ${ }^{*, \dagger}$ E. W. Meijer. ${ }^{\dagger}$
Laboratory of Macromolecular and Organic Chemistry, Eindhoven University of Technology, P.O. Box 513, 5600 MB Eindhoven, The Netherlands, Department of Materials, Eidgenössische Technische Hochschule Zürich, ETH Hönggerberg, CH-8093, Zürich, Switzerland, Department of Materials, Queen Mary, University of London, Mile End Road, London E1 4NS, United Kingdom, Danish
Polymer Centre, Risø National Laboratory, 4000 Roskilde, Denmark

\section{Supporting Information}

\section{Experimental Section}

Instrumentation. NMR spectra were recorded on a Varian Mercury $400 \mathrm{MHz}(100 \mathrm{MHz}$

for $\left.{ }^{13} \mathrm{C}\right)$ spectrometer or a Varian Gemini $500 \mathrm{MHz}\left(125 \mathrm{MHz}\right.$ for $\left.{ }^{13} \mathrm{C}\right)$ spectrometer. The chemical shifts $(\delta)$ are listed in parts per million (ppm) using residual non-deuterated solvent as internal standard $\mathrm{CDCl}_{3}\left({ }^{1} \mathrm{H}, \delta: 7.26 \mathrm{ppm}\right)$. High temperature proton NMR analysis of the copolymers was performed in 1,1,2,2-tetrachloroethane- $d_{2}$ at $140{ }^{\circ} \mathrm{C}$ and referenced to residual non-deuterated solvent peak $\left({ }^{1} \mathrm{H}, \delta: 6.0 \mathrm{ppm}\right)$. Matrix-assisted laser desorption/ionization time-of-flight (MALDI-TOF) mass spectrometry (MS) spectra were measured on a Perspective DE voyager spectrometer in linear mode with an accelerating voltage of 25,000 V, and a dithranol matrix. Molecular weights of the starting P3HT homopolymers and the P3HT-poly(cyclooctene) block copolymers were determined by 
gel permeation chromatography (GPC) using a Waters GPC equipped with a Shimadzu RID-10A refractive index detector and a Shimadzu SPD-M10A diode array detector at 25 ${ }^{\circ} \mathrm{C}$. Samples were run in HPLC grade THF at a flow rate of $1.0 \mathrm{~mL} \cdot \mathrm{min}^{-1}$ through two PLgel $5 \mu \mathrm{m}$ Mixed-C and Mixed-D columns and calibrated to external monodisperse polystyrene standards. GPC polymer solutions were prepared with a concentration of $c a$. $0.1 \% \mathrm{w} / \mathrm{v}$ in HPLC grade THF. Digital photographs were taken using a Canon Digital EOS 20D Camera using the autoexposure setting. Solutions used for photographs were prepared as $0.1 \mathrm{wt} \%$ in cyclohexanone and cooled at a rate of $1{ }^{\circ} \mathrm{C} / \mathrm{min}$. Thermal gravimetric analyses (TGA) were performed under an air atmosphere as indicated, at a heating rate of $5{ }^{\circ} \mathrm{C} / \mathrm{min}$ on a Mettler Toledo TGA STDA851 instrument. Differential scanning calorimetry (DSC) analyses of the polymers were recorded under a $\mathrm{N}_{2}$ atmosphere at a scan rate of $10{ }^{\circ} \mathrm{C} / \mathrm{min}$ on a Mettler Toledo DSC 822 instrument, with the temperature calibrated using an indium standard. The reported DSC curves are second heating and cooling scans, taken after an initial heating scan to erase thermal history. Grazing incidence wide angle X-ray scattering (WAXS) was performed using the Rigaku rotating anode setup at Risø National Laboratory. The wavelength was $\lambda=1.5418 \AA$ $\left(\mathrm{CuK}_{\alpha}\right)$, monochromatized by an X-ray multilayer mirror. To suppress background scattering from the substrate, the incoming beam was adjusted to hit the film at an angle of $\sim 0.18^{\circ}$. The scattered intensity was collected by a Fuji image plate mounted $12.0 \mathrm{~cm}$ from the sample. The chamber containing the film and the image plate was kept in vacuum during the experiment to reduce background scattering. The scattering vector is defined by $\mathbf{Q} \equiv \mathbf{k}_{\mathrm{f}}-\mathbf{k}_{\mathrm{i}}$, the difference between the in and outgoing wave vectors. The magnitude of $\mathbf{Q}$ is given by $|\mathbf{Q}|=4 \pi / \lambda \cos (\theta)$, where $\theta$ is half the total scattering angle $2 \theta$. 
Samples for WAXS analysis were taken from thin films prepared by dropcasting a 0.2 wt $\%$ solution of P3HT-PE in cyclohexanone onto a silicon wafer. UV-Vis spectra were recorded on a Perkin Elmer Lambda 900 spectrometer. Samples for UV-Visible spectroscopic measurements were prepared by dropcasting a $0.1 \mathrm{wt} \%$ solution of each polymer in xylenes.

Synthesis. All reactions were carried out under an atmosphere of argon. The reagents were purchased from Aldrich or Acros and were used as supplied unless otherwise stated. 2,5-Dibromo-3-hexyl thiophene and allyl-endcapped regioregular P3HT were synthesized according to literature procedures. ${ }^{1,2}$ The MALDI-TOF mass spectrum (Figure S1) confirmed the near-quantitative incorporation of the allyl end group. Regioregular poly(3-hexylthiophene) standard $\left(M_{\mathrm{n}}=14 \mathrm{~kg} \cdot \mathrm{mol}^{-1}, M_{\mathrm{w}}=22 \mathrm{~kg} \cdot \mathrm{mol}^{-1}\right)$ was generously supplied by Merck Chemicals. Polyethylene standard $\left(M_{\mathrm{n}}=100 \mathrm{~kg} \cdot \mathrm{mol}^{-1}, M_{\mathrm{w}}\right.$ $=119 \mathrm{~kg} \cdot \mathrm{mol}^{-1}$ ) was supplied by the U.S. Department of Commerce National Institute of Standards and Technology.

P3HT-Poly(cyclooctene) (P3HT-PCO) block copolymers. In a representative procedure, P3HT (0.050 g, $0.0066 \mathrm{mmol}$ allyl endgroup) and cyclooctene $(0.100 \mathrm{~g}, 0.91$ mmol) were dissolved in $2 \mathrm{~mL}$ of chlorobenzene, which was previously degassed by three freeze-pump-thaw cycles. The solution was purged with argon for $c a .15$ min, after which $0.20 \mathrm{~mL}$ of a stock solution of the catalyst $\left(1 \mathrm{mg} \cdot \mathrm{mL}^{-1}\right.$ in chlorobenzene) was added to the reaction mixture. The solution was stirred at $55{ }^{\circ} \mathrm{C}$ for 24 hours. The polymer was then precipitated into methanol, filtered and directly submitted for hydrogenation.

P3HT-Polyethylene (P3HT-PE) block copolymers. In a typical procedure, $0.143 \mathrm{~g}$ of P3HT-PCO was suspended in $20 \mathrm{~mL} p$-xylene with $p$-toluenesulfonhydrazide 
(0.97 g, $5.2 \mathrm{mmol})$ (ca. 6 equivalents per cyclooctene olefin) and a few crystals of BHT. The mixture was thoroughly degassed and stirred under an argon atmosphere. Triethylamine $(0.53 \mathrm{~g}, 5.2 \mathrm{mmol})$ was added to the stirring mixture. After approximately 8 hours at $130^{\circ} \mathrm{C}$, the solution was precipitated into $150 \mathrm{~mL}$ of stirring methanol to yield a purple solid. The solid was filtered through a soxhlet thimble, and purified by soxhlet extraction with methanol, THF and chloroform to remove impurities and unreacted P3HT. The P3HT-PE block copolymer was extracted with $p$-xylene and reprecipitated into methanol. The solid was collected and characterized by NMR in order to determine the weight percentage and molecular weight of the copolymers. We note that this synthetic strategy theoretically does not preclude the formation of some triblock copolymers. However, we expect that we are preparing primarily diblock copolymers as the catalyst:P3HT chain transfer agent ratio is high. ${ }^{3,4}$ Yield: $45 \% .{ }^{1} \mathrm{H}-\mathrm{NMR}$ (at $140{ }^{\circ} \mathrm{C}$ ) (1,1,2,2-tetrachloroethane, $500 \mathrm{MHz}), \delta: 7.06,4.15\left(\mathrm{CH}_{2}, \mathrm{~m}\right), 2.90\left(\mathrm{CH}_{2}, \mathrm{t}\right), 1.81\left(\mathrm{CH}_{2}\right.$, $\mathrm{m}), 1.10-1.65\left(\mathrm{CH}_{2-P 3 H T}, \mathrm{CH}_{2-P E}, \mathrm{~m}\right), 1.01(3 \mathrm{H}, \mathrm{t})$.

Elemental analysis for polymers $1(81.73 \% \mathrm{C}, 13.11 \% \mathrm{H}, 2.60 \% \mathrm{~S})$ and $3(80.73 \% \mathrm{C}$, $12.89 \% \mathrm{H}, 3.35 \% \mathrm{~S}$ ) provide an estimated content of 13.8 and $17.9 \mathrm{wt} \% \mathrm{P} 3 \mathrm{HT}$ in the block copolymer, in agreement with estimates from NMR (Table S1).

Peak molecular weights determined by GPC for the three block copolymers are: for 1: $M_{\mathrm{p}}$ $=101.9 \mathrm{~kg} \mathrm{~mol}^{-1} ; 2: M_{\mathrm{p}}=88.3 \mathrm{~kg} \mathrm{~mol}^{-1} ;$ and $3: M_{\mathrm{p}}=61.4 \mathrm{~kg} \mathrm{~mol}^{-1}$; 
Table S1. Thermal DSC Data for P3HT-PE Block Copolymers

\begin{tabular}{ccccccc}
\hline Polymer & $\begin{array}{c}\text { P3HT } \\
\text { wt. \% }\end{array}$ & $\begin{array}{c}\mathrm{T}_{\mathrm{m}} 1 /{ }^{\circ} \mathrm{C} \\
\left(\Delta \mathrm{H} / \mathrm{Jg}^{-1}\right)\end{array}$ & $\begin{array}{c}\mathrm{T}_{\mathrm{c}} 1 /{ }^{\circ} \mathrm{C} \\
\left(\Delta \mathrm{H} / \mathrm{Jg}^{-1}\right)\end{array}$ & $\begin{array}{c}\mathrm{T}_{\mathrm{m}} 2 /{ }^{\circ} \mathrm{C} \\
\left(\Delta \mathrm{H} / \mathrm{Jg}^{-1}\right)\end{array}$ & $\begin{array}{c}\mathrm{T}_{\mathrm{c}} 2 /{ }^{\circ} \mathrm{C} \\
\left(\Delta \mathrm{H} / \mathrm{Jg}^{-1}\right)\end{array}$ & $\begin{array}{c}\text { Mol.Wt. } \\
\left(\mathrm{kg} \mathrm{mol}^{-1}\right)\end{array}$ \\
\hline PE & $0 \%$ & $132(181)$ & $117(-181)$ & & & 100 \\
$\mathbf{1}$ & $11 \%{ }^{\mathrm{a}}$ & $131(139)$ & $112(-143)$ & $214(1.55)$ & $--^{\mathrm{c}}$ & $142^{\mathrm{b}}$ \\
$\mathbf{2}$ & $14 \%^{\mathrm{a}}$ & $129(133)$ & $108(-140)$ & $214(1.83)$ & $161(-1.42)$ & $109^{\mathrm{b}}$ \\
$\mathbf{3}$ & $22 \%{ }^{\mathrm{a}}$ & $128(114)$ & $107(-124)$ & $219(3.16)$ & $181(-2.95)$ & $71^{\mathrm{b}}$ \\
P3HT & $100 \%$ & & & $221(24.0)$ & $195(-21.5)$ & 14 \\
\hline
\end{tabular}

${ }^{a}$ Weight percentages of P3HT in copolymers 1-3 were determined by the integration of P3HT resonances relative to PE resonance. ${ }^{b}$ Molecular weights based on $M_{\mathrm{n}}$ (GPC) of the starting P3HT. ${ }^{\mathrm{c}}$ Not observed.
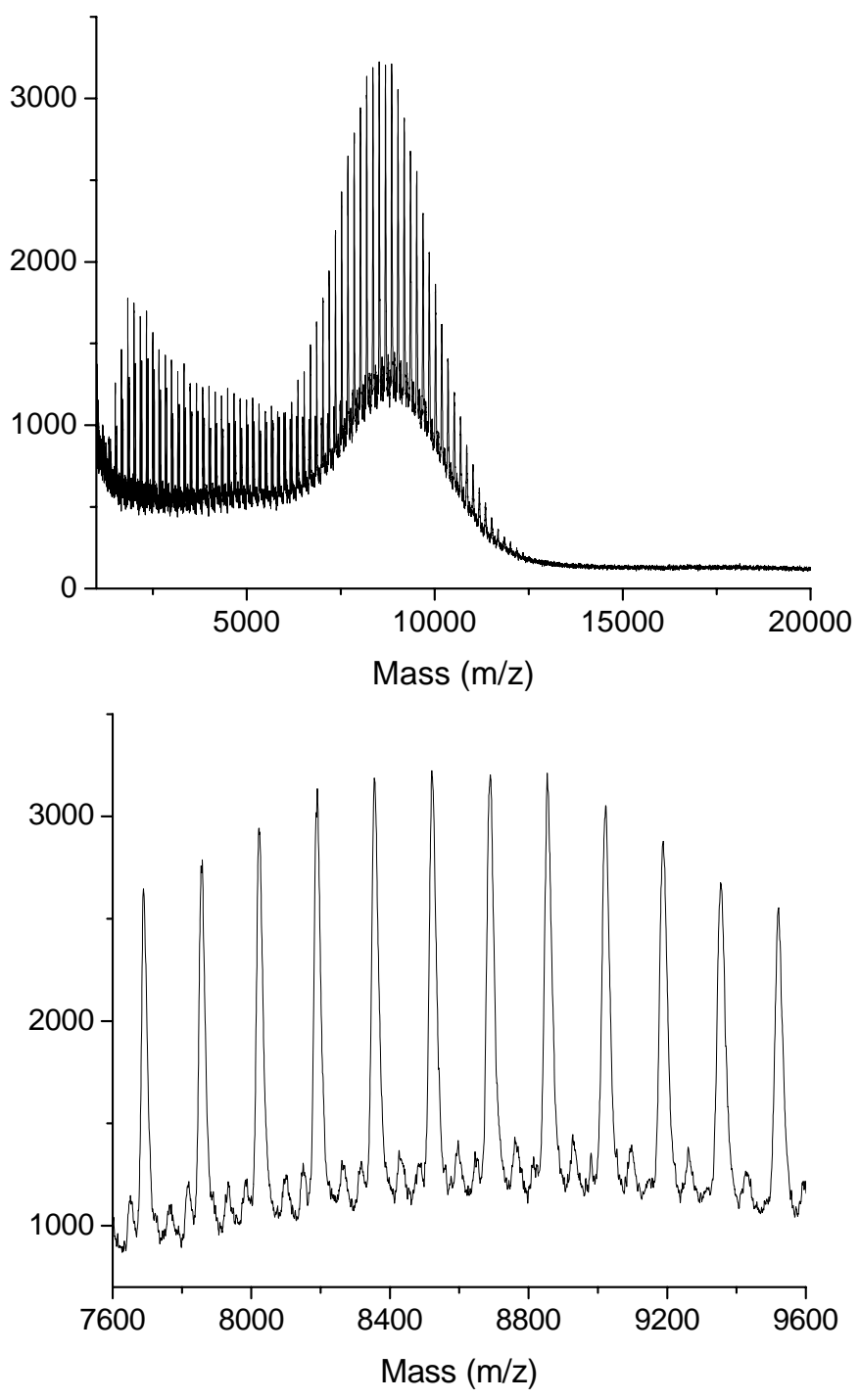

Figure S1 MALDI-TOF mass spectrum (top) and enlargement (bottom) of allyl functionalized P3HT . 


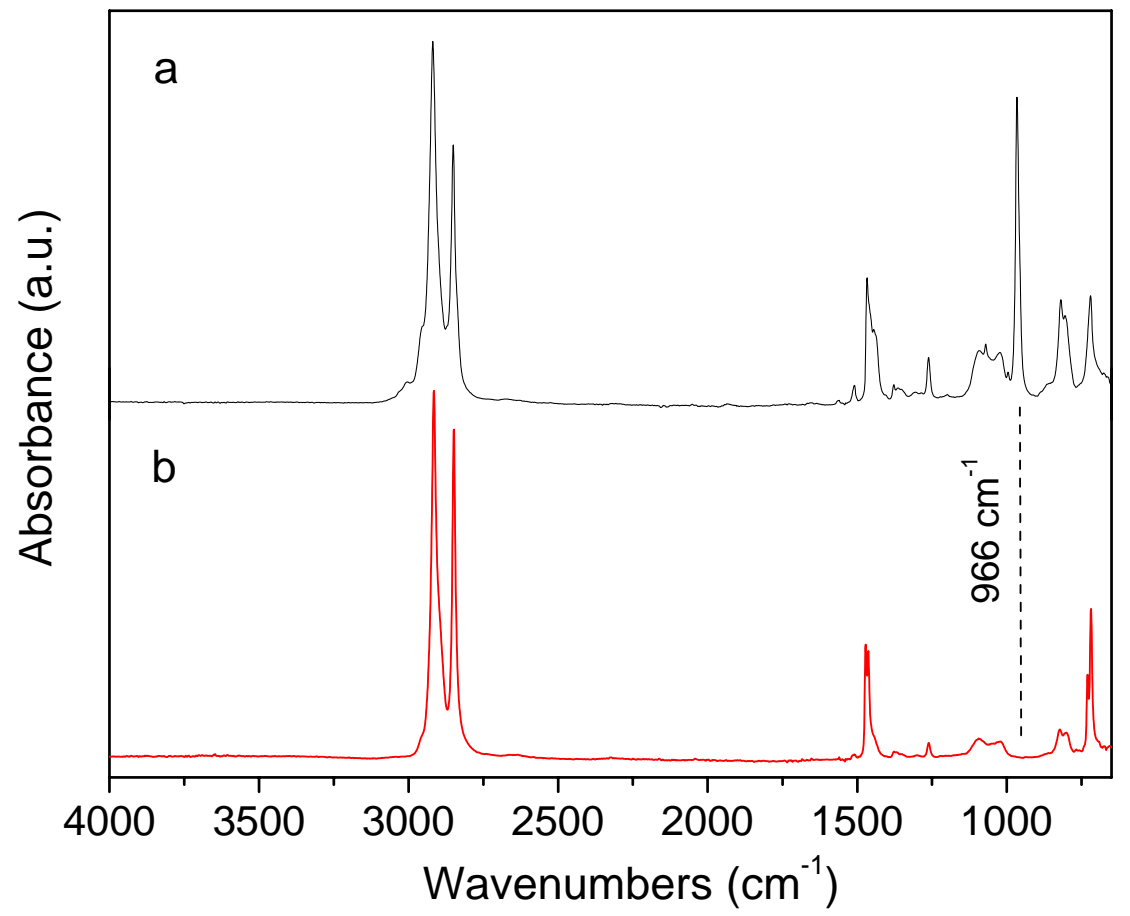

Figure S2. FT-IR spectrum of (a) P3HT-PCO and (b) P3HT-PE. Exhaustive hydrogenation of the PCO block is confirmed by the disappearance of the characteristic alkene $\mathrm{C}-\mathrm{H}$ bend $\left(966 \mathrm{~cm}^{-1}\right)$ which is present in the unsaturated block.

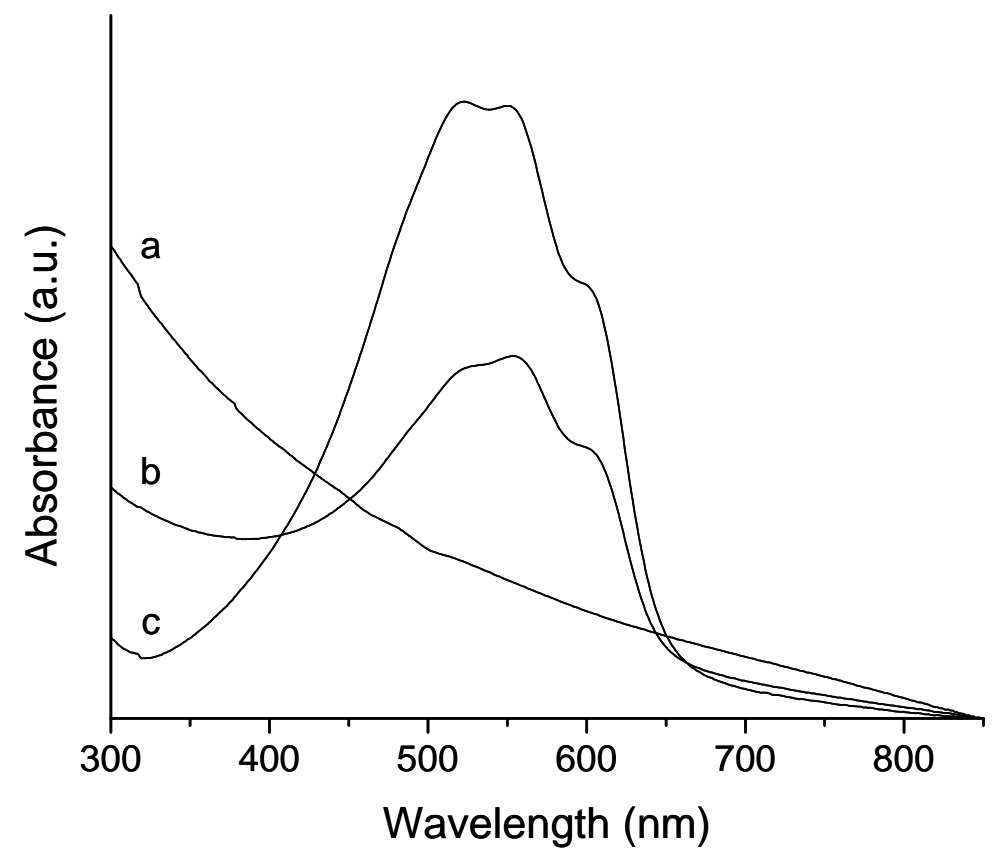

Figure S3. UV-Visible spectrum of (a) PE, (b) P3HT-PE and (c) P3HT as thin films at room temperature on glass, cast from $0.1 \mathrm{wt} \%$ solutions in xylene. 


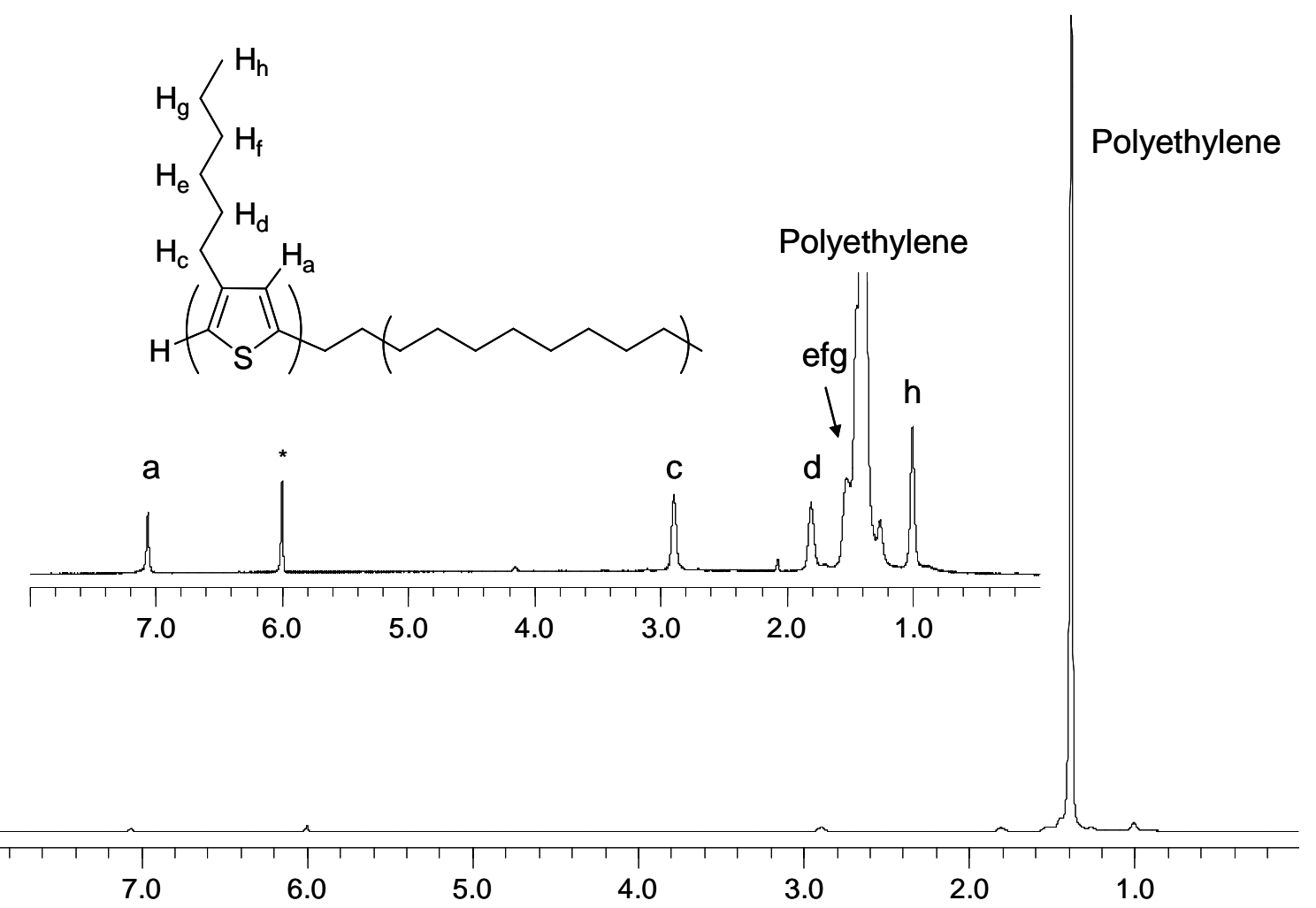

Figure S4. ${ }^{1} \mathrm{H}$ NMR spectrum of P3HT-PE in 1,1,2,2-tetrachloroethane $(*)$.

1 Loewe, R. S.; Khersonsky, S. M.; McCullough, R. D. Adv. Mater. 1999, 11, 250253.

2 Jeffries-El, M.; Sauve, G.; McCullough, R. D. Adv. Mater. 2004, 16, 1017-1019.

3 Scherman, O. A.; Rutenberg, I. M.; Grubbs, R. H. J. Am. Chem. Soc. 2003, 125, 8515-8522.

4 Hillmyer, M. A.; Grubbs, R. H. Macromolecules 1995, 28, 8662-8667. 